\title{
Observation of the critical regime near Anderson localization of light
}

\author{
Martin Störzer, Peter Gross, Christof M. Aegerter, and Georg Maret \\ Fachbereich Physik, University of Konstanz, Germany
}

(October 3, 2018)

\begin{abstract}
Diffusive transport is among the most common phenomena in nature [1]. However, as predicted by Anderson [2], diffusion may break down due to interference. This transition from diffusive transport to localization of waves should occur for any type of classical or quantum wave in any media as long as the wavelength becomes comparable to the transport mean free path $\ell^{*}[3]$. The signatures of localization and those of absorption, or bound states, can however be similar, such that an unequivocal proof of the existence of wave localization in disordered bulk materials is still lacking. Here we present measurements of time resolved non-classical diffusion of visible light in strongly scattering samples, which cannot be explained by absorption, sample geometry or reduction in transport velocity. Deviations from classical diffusion increase strongly with decreasing $\ell^{*}$ as expected for a phase transition. This constitutes an experimental realization of the critical regime in the approach to Anderson localization.
\end{abstract}

Diffusive transport controls many phenomena in biology, chemistry, physics and engineering, where undirected transport is characterized by a linear increase of the mean square displacement $\left\langle r^{2}\right\rangle$ with time [1]. As a consequence, transmission of particles through a slab of length $L$ is proportional to $\ell^{*} / L$ known in the context of electrons in metals as Ohm's law. However, when the wave-nature of the diffusing particles is taken into account, constructive interference of waves propagating on reciprocal multiple scattering paths may lead to a breakdown of diffusion and the particles are "trapped" on closed multiple scattering loops. This means that the probability of returning to the starting point of such a closed loop is increased twofold due to the fact that the path has the same length in both counter-propagating directions. Thus diffusive transport away from this starting point is reduced. Anderson first predicted this destruction of diffusion in 1958 [2] to explain the metal-insulator transition. If the scattering power of the medium is high, i.e. the transport mean free path $\ell^{*}$ is small, the density of closed loops increases which leads to a reduced transport inside the material. This can be described by a rescaling of the diffusion coefficient [4]. For strong localization the rescaled diffusion coefficient becomes zero at finite length scales, such that the spread of $\left\langle r^{2}\right\rangle$ comes to an end at a length scale corresponding to the localization length. The transition from a diffusive to a localized state should occur when the wavelength $\lambda$ becomes comparable to $\ell^{*}$, as quantified by the Ioffe-Regel criterion $k \ell^{*} \simeq 1$ where $k=2 \pi / \lambda$ is the wavenumber [3].

While localization of electrons in metals was widely studied [5], an unequivocal demonstration of the pure interference effect as predicted by Anderson turned out to be difficult in this case, as electrons bound in deep minima of a random potential are virtually impossible to distinguish from those in closed loops. However, photons in disordered materials revealed an excellent model system to study localization effects $[6,7]$ : In contrast to elec- trons, they do not interact with each other, nor can they be bound in a random potential. Hence all deviations from classical diffusion have to be due to interference, when absorption is taken into account properly. Moreover, in order to create a strongly scattering medium, materials having a large optical refractive index combined with very small absorption are available for visible light.

Thus there have been many investigations in the past studying diffusive transport of visible $[8,9]$ and infrared light [10]. For visible light, where reported values of $k \ell^{*}$ go down to 3.2 [8], measurements have mainly focused on static transmission experiments where a decay faster than $\propto 1 / L$ was interpreted as evidence for the onset of localization [12]. It was noted however that absorption, which is always present in such samples, can lead to a similar decay $[13,14]$. Furthermore, recent investigations of time resolved transmission of these samples with similar values of $k \ell^{*}$ show no discernible deviation from purely diffusive behavior [9]. Localization of photons has also been studied by use of microwave scattering in disordered media [14-16], where indications of a decreasing diffusion coefficient have been found [16]. However it has to be noted that these investigations were carried out in tubes of extent $\sim 2 \times 2 \times 20$ in units of $\ell^{*}$ [17], which constitute a relatively small quasi one-dimensional (1D) system. As localization is always present in 1D and 2D [4], theoretical descriptions of the quasi 1D geometry $[17,18]$ indicate that observations of localization effects are possible far away from $k \ell^{*} \simeq 1$. Similar theories in 3D [19] find a negligible effect in the same regime. Thus, an unequivocal proof of the transition to localization in three dimensions is still lacking.

Here we present measurements of time resolved photon transport through bulk powders of $\mathrm{TiO}_{2}$ with typical sample dimensions of $\sim 10^{5} \times 10^{5} \times 10^{4}$ in units of $\ell^{*}$. These measurements have the advantage that they allow a direct determination of the photon path length dependence of the diffusion coefficient, where absorption and 
localization lead to different functional forms of the tail (exponential vs. non-exponential) of the path length distribution [18]. Furthermore we independently measure the turbidity of the samples using coherent backscattering $[20,21]$. This allows a systematic study of the approach to the localization transition as a function of turbidity $\left(k \ell^{*}\right)^{-1}$.

Our samples consist of ground $\mathrm{TiO}_{2}$ particles in its rutile structure with a refractive index of 2.8. The resulting grains of the different samples have various average particle sizes with average diameters ranging from $220 \mathrm{~nm}$ to $550 \mathrm{~nm}$ with a polydispersity of $\sim 25 \%$. Those particles are commercially available as pigments for white paint from e.g. DuPont or Aldrich. In order to minimize $\ell^{*}$ these powders are compressed to obtain filling fractions of $\phi \simeq 0.4$. The scattering properties are determined from coherent backscattering [20,21]. The angular width of this enhancement to the incoherent background is inversely proportional to $k \ell^{*}[22,23]$. For the highly scattering samples studied here, the width becomes very broad, which is why the distribution has to be measured to very wide angles for a good determination of the incoherent background. Our setup was custom designed for this problem (see methods) [24].

Fig.1 shows the backscattering angular distribution for two different samples, S1 and S3, corresponding to average particle diameters of $250 \mathrm{~nm}$ and $550 \mathrm{~nm}$ respectively. Using the value of the averaged refractive index from numerical simulations based on the energy density coherent potential approximation [25], we determine the reflectivity of the surface and are hence able to correct for the overestimation of $k \ell^{*}$ due to internal reflections [26]. Thus the values of $k \ell^{*}$ are determined from the full widths at half maximum of the curves with a correction for the reflectivity of the surface. This gives values ranging from $k \ell^{*}=6.3$ for $\mathrm{S} 3$ to $k \ell^{*}=2.5$ for $\mathrm{S} 1$ at a wavelength of $590 \mathrm{~nm}$, close to the Ioffe-Regel criterion.

In order to measure the time resolved transmission we use a single photon counting method, where the time delay of a picosecond light pulse transmitted through the sample is measured. From a histogram of time of flights of many such pulses, the path length distribution inside the sample is obtained directly $[9,27,28]$. Our setup consists of a dye laser working at a wavelength of $590 \mathrm{~nm}$ with a pulse width of $\sim 20$ ps. The time of flight histograms have been deconvoluted with the pulse shape of the laser system in order to recover the pure path length distributions (see methods). Fig. 2 shows the path length distributions in the samples characterized in Fig. 1 compared to another sample (S2) with $k \ell^{*}=4.3$. As can be seen in Fig. 2A, classical diffusion theory including absorption $[29,30]$ fits the data from sample S3 very well. In contrast, the path length distribution of S1 (Fig. 2C) shows marked deviations from diffusive behaviour. In particular, we observe a non-exponential decay at long times, with photons staying inside the sample longer than expected from a purely diffusive process. In Fig. 2B, the data from sample S2 show small deviations from the diffusion picture.

Diffusion in an extended slab of thickness $L$, which in our experiments ranges from $1.3 \mathrm{~mm}$ to $2.5 \mathrm{~mm}$, leads to an exponential decay of the time dependent transmission at long times as $I(t) \propto e^{-\left(\frac{\pi^{2} D(t)}{L^{2}}+\frac{c}{n \ell_{a}}\right) t}[29,30]$. Here $\ell_{a}$ is the absorption length, which in our samples ranges from $0.3 \mathrm{~m}$ to $2.6 \mathrm{~m}, c$ is the speed of light in vacuum, $n$ is the effective refractive index of the medium, and $D$ the diffusion coefficient. Note that the values for $\ell_{a}$ are more than a factor of $10^{6}$ larger than typical values of $\ell^{*}$ in our samples. Any non-exponential decay of $I(t)$ at long times thus indicates a temporally varying diffusion coefficient $D(t)$. Using the properties of $I(t)$ at long times, we can obtain a direct measurement of the diffusion coefficient up to a constant given by $\ell_{a}$. This is achieved by taking the negative time derivative of the logarithm of the intensity divided by $\left(\pi^{2} D(t=0) / L^{2}+c / n \ell_{a}\right)$, where $\pi^{2} D(t=0) / L^{2}$ is the inverse of the time $t_{\max }$, at which most photons leave the sample [16]. This is shown in Fig. 3 for samples S1, S2, and S3. For long times the classical sample S3 approaches the constant value of 1 , whereas the curve for S1 shows a strongly decreasing diffusion coefficient and S2 reveals intermediate behaviour.

To quantify the non-classicality of the diffusive light transport through the different samples we determined the average of the ratio of the measured data to the diffusion fits. This was done systematically for all powders over the time interval from $t_{\max }$ to $3 t_{\max }$. This deviation is given as a function of $k \ell^{*}$ in Fig. 4. The figure clearly shows that the deviations increase strongly when approaching the Ioffe-Regel criterion, as expected for a phase transition.

The data we have presented show clear deviations from diffusive transport through these highly scattering samples. The non-exponential decay of the path length distribution indicates a renormalized value of the diffusion coefficient at long times as was predicted by scaling theory $[4,7]$. Moreover, the deviations from diffusive behaviour scale with the value of $k \ell^{*}$ consistent with the IoffeRegel criterion [3] and the approach to a phase transition. These deviations cannot be explained by absorption as this only leads to an additional exponential decrease but cannot introduce a non-exponential path length distribution. There is also no systematic dependence of the values of the absorption length of the various samples on $k \ell^{*}$ in contrast to the monotonic dependence of the deviations from classical diffusion. A change in the transport velocity due to resonant scattering [31] can also be ruled out, since values of the diffusion coefficient from the classical fits, such as that in Fig. 2C, are consistent with a value of the transport velocity of $c / n$. Furthermore such a change is not expected to be dependent on the length of the specific path and hence would not lead to a 
non-exponential decay as the data show. Finally, sample inhomogeneities, such as a stratification, cannot account for the effect, as the path length distributions are found to be independent of the direction of illumination. We thus conclude that these observations constitute direct evidence for the slowing down of photon diffusion due to the approach to the Anderson localization transition and hence for the existence of the transition to strong localization of photons in three dimensions.

\section{METHODS}

\section{Coherent backscattering}

The diffuse reflected intensity is not constant but depends on the backscattering angle $\theta$ as $\cos (\theta)$, such that the difference of the backscattering signal from this functional shape has to be determined. Our setup consists of 256 photo-sensitive diodes attached to an arc with a diameter of $1.2 \mathrm{~m}$ in order to get sufficient angular resolution over a range of $-70^{\circ}<\theta<+70^{\circ}[24]$. Here, the resolution is $1^{\circ}$ for $|\theta|>20^{\circ}, 0.7^{\circ}$ for $10^{\circ}<|\theta|<20^{\circ}$ and $0.14^{\circ}$ for $|\theta|<10^{\circ}$. In addition, the central part of the backscattering cone, $-3^{\circ}<\theta<+3^{\circ}$ was measured separately using a beam-splitter and a charged coupled device camera to a resolution of $0.02^{\circ}$. The measurements are done using circularly polarized light in order to reduce the influence of singly scattered light.

\section{Time of flight measurements}

Our setup consists of a dye laser working at a wavelength of $590 \mathrm{~nm}$ with a pulse width of $\sim 20 \mathrm{ps}$. The Rhodamin6G dye laser (Coherent C699) is pumped by an $\mathrm{Ar}^{+}$laser (Coherent Inova400) and was modified by APE with a mode locker and a cavity dumper to deliver picosecond pulses. In order to recover the pure path length distributions, the time of flight histograms have to be deconvoluted with the pulse shape of the laser system. This is because after pulses, albeit strongly suppressed, and indiscriminate noise may lead to disturbances in the time of flight measurements. For this purpose, we measured pulse shape, including the noise level, in the absence of a sample. This zero-pulse was then deconvoluted with the time of flight data in Fourier space to directly give the path length distribution for a supposed delta-peaked pulse as it is calculated theoretically.

The observed non-exponential decay is not due to a distribution of $\ell^{*}$ values in the sample due to e.g. stratification. We have confirmed this by measuring the time of flight distributions with sample S1 flipped, such that the direction of stratification would be reversed. These measurements show the same non-exponential decay as Fig. 2C.

\section{ACKNOWLEDGEMENTS}

This work was supported by the Deutsche Forschungsgemeinschaft, the International Research and Training Group "Soft Condensed Matter of Model Systems" and the Center for Applied Photonics (CAP) at the University of Konstanz. Furthermore, we would like to thank DuPont chemicals and Aldrich for providing samples used in this study.

[1] Einstein, A., Über die von der molekularkinetischen Theorie der Wärme geforderte Bewegung von in ruhenden Flüssigkeiten suspendierten Teilchen. Ann. Phys. 17, 549 (1905).

[2] Anderson, P.W., Absence of diffusion in certain random lattices. Phys. Rev. 109, 1492 (1958).

[3] Ioffe, A.F. and Regel, A.R., Non-crystalline, amorphous and liquid electronic semiconductors. Progress in Semiconductors 4, 237 (1960).

[4] Abrahams, E., Anderson, P.W., Licciardello, D. and Ramakrishnan, T.V., Scaling theory of localization: absence of quantum diffusion in two dimensions. Phys. Rev. Lett. 42, 673 (1979).

[5] Altshuler, B.L., Lee, P.A., Webb, R.A., Eds, Mesoscopic Phenomena in Solids (North-Holland, Amsterdam, 1991).

[6] Anderson, P.W., The question of classical localization: a theory of white paint? Phil. Mag. B 52, 505 (1985).

[7] John, S., Strong Localization of Photons in Certain Disordered Dielectric Superlattices. Phys. Rev. Lett. 58, 2486 (1987).

[8] Schuurmans, F.J.P., Megens, M., Vanmaekelbergh, D., and Lagendijk, A., Light scattering near the localization transition in macroporous GaP networks. Phys. Rev. Lett. 83, 2183 (1999).

[9] Johnson, P.M., Imhof, A., Bret, B.P.J., Gómez Rivas, J., and Lagendijk, A., Time resolved pulse propagation in a strongly scattering material, Phys. Rev. E 68, 016604 (2003).

[10] Gómez Rivas, J., Sprik, R., Lagendijk, A., Noordam, L.D., and Rella, C.W., Mid-infrared scattering and absorption in Ge powder close to the Anderson localization transition. Phys. Rev. E 62, R4540 (2000).

[11] Gómez Rivas, J., Sprik, R., Lagendijk, A., Noordam, L.D., and Rella, C.W., Static and dynamic transport of light close to the Anderson localization transition. Phys. Rev. E 63, 046613 (2001).

[12] Wiersma, D.S., Bartolini, P., Lagendijk, A., and Righini, R., Localization of light in a disordered medium. Nature 390, 671 (1997). 
[13] Scheffold, F., Lenke, R., Tweer, R., and Maret, G., Localization or classical diffusion of light? Nature 398, 206 (1999); see also Wiersma, D.S., Gómez Rivas, J., Bartolini, P., Lagendijk, A., and Righini, R., reply Nature 398, 207 (1999).

[14] Chabanov, A.A., Stoytchev, M., and Genack, A.Z., Statistical signatures of photon localization. Nature 404, 850 (2000).

[15] Chabanov, A.A. and Genack, A.Z., Statistics of Dynamics of Localized Waves. Phys. Rev. Lett. 87, 153901 (2001).

[16] Chabanov, A.A., Zhang, Z.Q., and Genack, A.Z., Breakdown of Diffusion in Dynamics of Extended Waves in Mesoscopic Media. Phys. Rev. Lett. 90, 203903 (2003).

[17] Cheung, S. K., Zhang, X., Zhang, Z. Q., Chabanov, A. A., and Genack, A. Z., Impact of Weak Localization in the Time Domain, Phys. Rev. Lett. 92, 173902 (2004).

[18] Skipetrov, S.E. and van Tiggelen, B.A., Dynamics of Weakly Localized Waves. Phys. Rev. Lett. 92, 113901 (2004).

[19] Skipetrov, S.E. and van Tiggelen, B.A., Dynamics of Anderson localization in open 3D media. cond-mat/0508726.

[20] Van Albada, M.P. and Lagendijk, A., Observation of weak localization of light in a random medium. Phys. Rev. Lett. 55, 2692 (1985).

[21] Wolf, P.E. and Maret, G., Weak localization and coherent backscattering of photons in disordered media. Phys. Rev. Lett. 55, 2696 (1985).

[22] Akkermans, E., Wolf, P.E., and Maynard, R., Coherent Backscattering of Light by Disordered Media: Analysis of the Peak Line Shape. Phys. Rev. Lett. 56, 1471 (1986).

[23] van Tiggelen, B.A., Lagendijk, A., and Wiersma, D.S., Reflection and transmission of waves near the localization threshold. Phys. Rev. Lett. 84, 4333 (2000).

[24] Gross, P., Diploma Thesis, University of Konstanz (2005).

[25] Busch, K. and Soukoulis, C.M., Transport properties of random media: An energy-density CPA approach. Phys. Rev. B 54, 893 (1996).

[26] Zhu, J.X., Pine, D.J., and Weitz, D.A., Internal reflection of diffusive light in random media. Phys. Rev. A 44, 3948 (1991).

[27] Watson, G.H., Fleury, P.A., and McCall, S.L., Searching for photon localization in the time domain. Phys. Rev. Lett. 58, 945 (1987).

[28] Drake, J.M., Genack, A.Z., Observation of nonclassical optical diffusion. Phys. Rev. Lett. 63, 259 (1989).

[29] Lenke, R. and Maret, G., Multiple Scattering of Light: Coherent Backscattering and Transmission in Scattering in Polymeric and Colloidal Systems, Brown, W., Mortensen, K., Eds. (Gordon and Breach Scientific, New York, 2000), chap 1.

[30] Berkovitz, R. and Kaveh, M., Propagation of waves through a slab near the Anderson transition: a local scaling approach. J. Phys. C: Cond. Mat. 2, 307 (1990).

[31] van Albada, M.P., van Tiggelen, B.A., Lagendijk, A., and Tip, A., Speed of propagation of classical waves in strongly scattering media. Phys. Rev. Lett. 66, 3132 (1991).

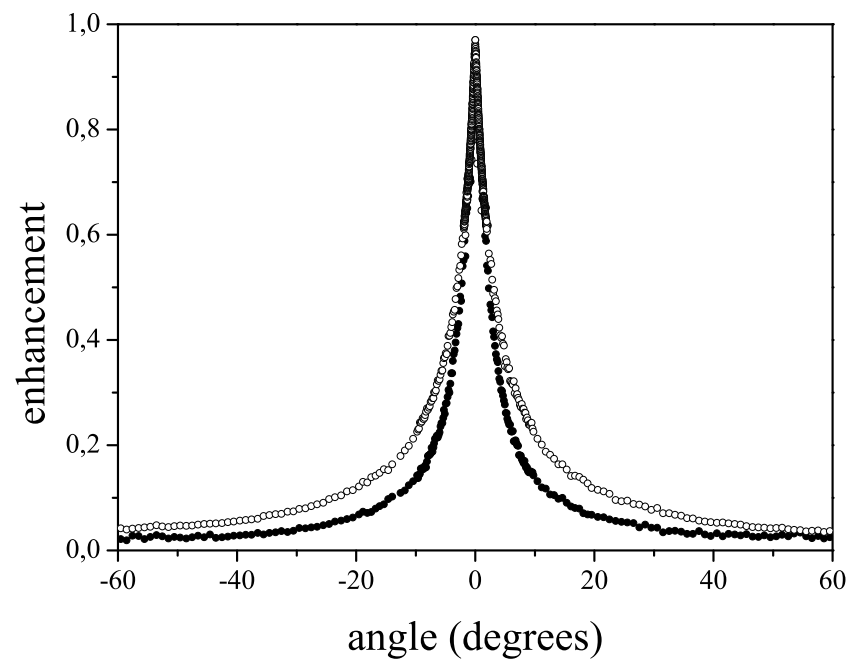

FIG. 1. Measurements of coherent backscattering for two different samples. Open symbols: sample S1 with an average particle diameter of $250 \mathrm{~nm}$ which yields $k \ell^{*}=2.5$. Closed symbols: sample S3 with an average diameter of $550 \mathrm{~nm}$ and $k \ell^{*}=6.3$. All measurements were done with circularly polarized light at a wavelength $2 \pi / k=590 \mathrm{~nm}$. 


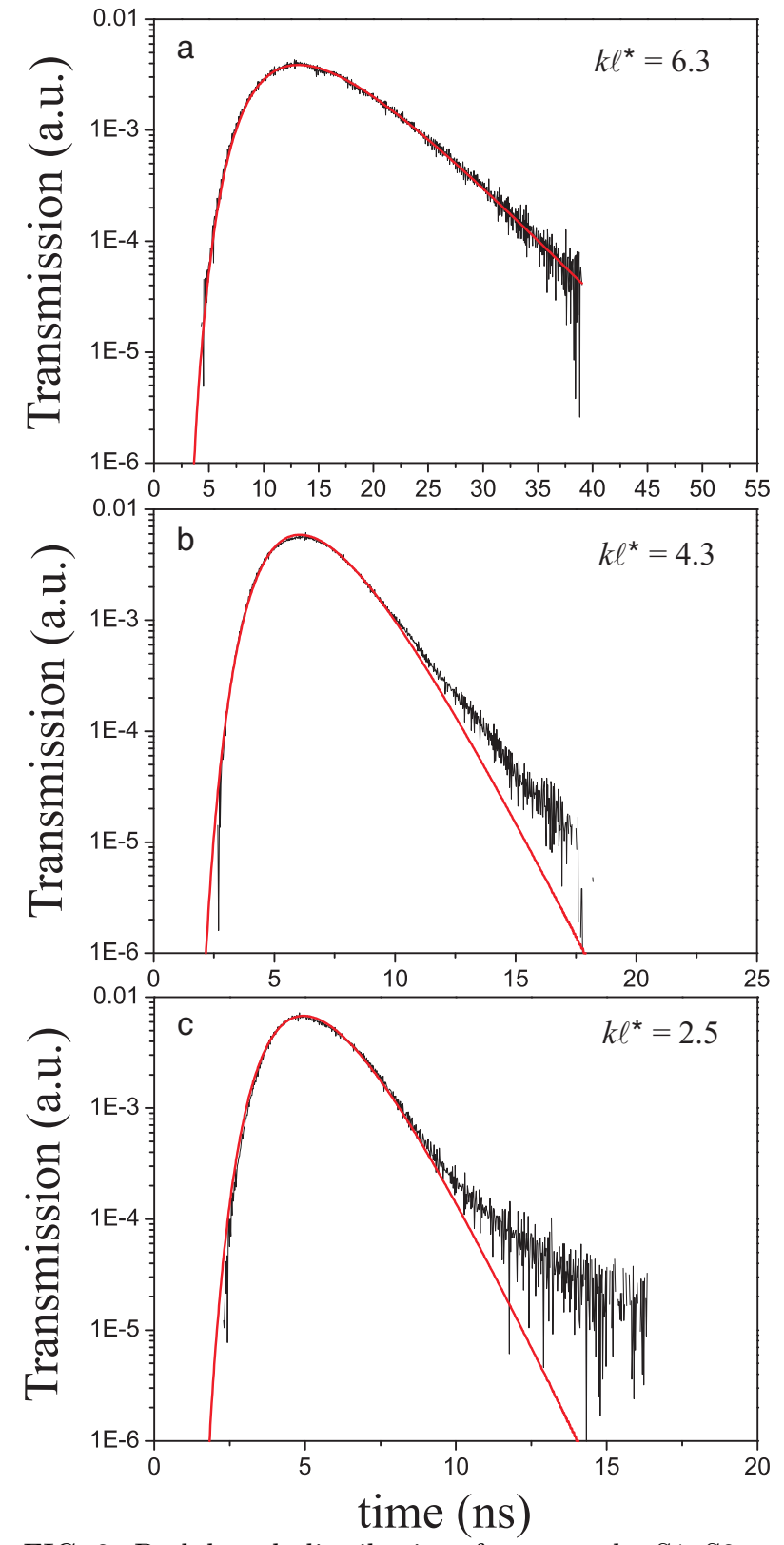

FIG. 2. Path length distributions from samples S1, S2, and S3. The experimental results are compared to diffusion theory including absorption. In $\mathrm{A}$, one can see that the data from S3 $\left(L=2.5 \mathrm{~mm}, D=22 \mathrm{~m}^{2} / \mathrm{s}, \ell_{a}=2600 \mathrm{~mm}\right)$ closely follow the diffusion fit, showing an exponential decay at long times. Part $\mathrm{C}$ in contrast shows strong deviations from the diffusion fit for $\mathrm{S} 1\left(L=1.48 \mathrm{~mm}, D=15 \mathrm{~m}^{2} / \mathrm{s}, \ell_{a}=340 \mathrm{~mm}\right)$, with a clearly non-exponential decay at long times. These deviations can be explained by a time dependent diffusion coefficient in the sample. An intermediate case is shown in part B from sample S2 $\left(L=1.51 \mathrm{~mm}, D=13 \mathrm{~m}^{2} / \mathrm{s}, \ell_{a}=380 \mathrm{~mm}\right)$, with a value of $k \ell^{*}=4.3$, where small deviations from the classical behaviour can be observed.

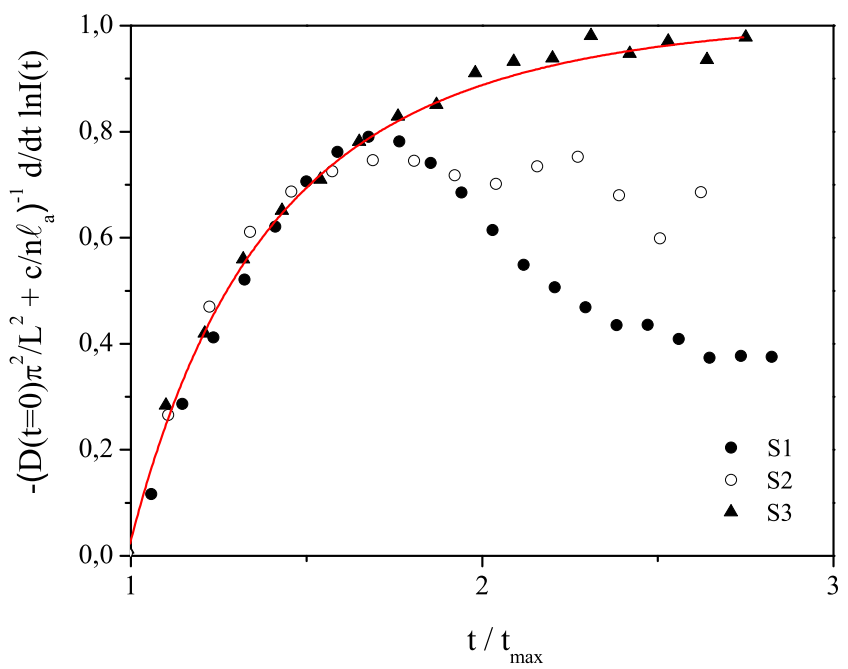

FIG. 3. Taking the negative derivative of the logarithm of the measured data one obtains a measure of the time dependence of the diffusion coefficient out of the time of flight data. Sample S3 has a constant diffusion coefficient at long times in agreement with diffusion theory indicated by the solid line. S1 and S2 in contrast show a decay illustrating the decrease of the diffusion coefficient of light with time. This demonstrates the existence of localized modes.

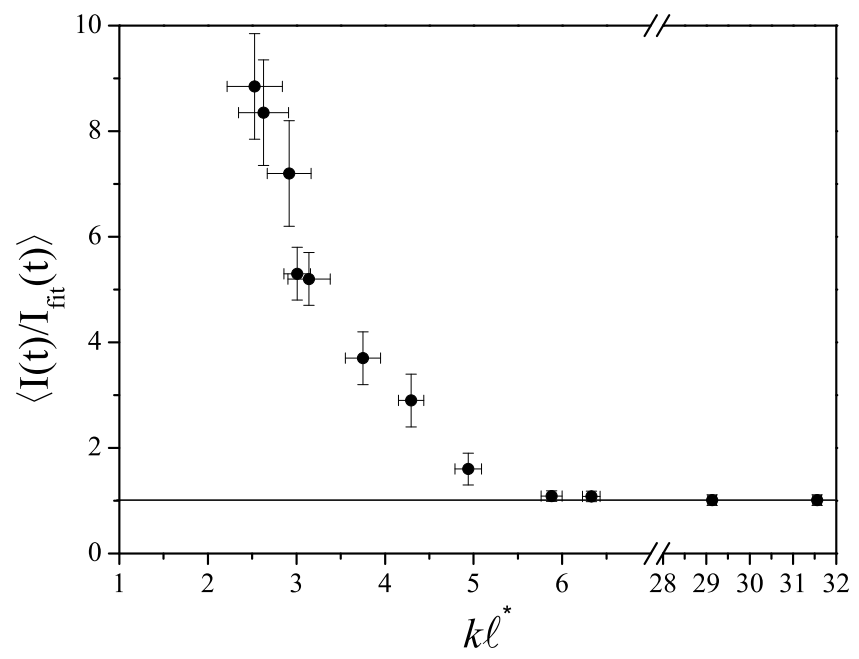

FIG. 4. Measurements of the deviations from diffusion on several samples with different values of $k \ell^{*}$. The deviation is quantified by taking the ratio of the experimental path length distribution, $I(t)$, to the classical fit, $I_{f i t}(t)$, and averaging it over the time interval from $t_{\max }$ to $3 t_{\max }$. The results were plotted over the $k \ell^{*}$ value determined from coherent backscattering (see text). Samples that have a $k \ell^{*}$ nearer to the Ioffe-Regel criterion show a bigger deviation from diffusive behaviour. 